\title{
PhenX: Vector measures for tobacco regulatory research
}

\section{Kurt M Ribisl, ${ }^{1}$ Frank J Chaloupka, ${ }^{2}$ Thomas R Kirchner, ${ }^{3}$ Lisa Henriksen, ${ }^{4}$ Destiney S Nettles, ${ }^{5}$ Rebecca C Geisler, ${ }^{5}$ Tabitha P Hendershot, ${ }^{5}$ Gary E Swan, ${ }^{4}$ PhenX TRR Vector Working Group}

${ }^{1}$ Health Behavior, UNC Gillings School of Global Public Health, Chapel Hill, North Carolina, USA ${ }^{2}$ Economics, University of Illinois at Chicago, Chicago, Illinois, USA

${ }^{3}$ College of Global Public Health, New York University, New York City, New York, USA ${ }^{4}$ Stanford Prevention Research Center, Stanford University School of Medicine, Stanford, California, USA

${ }^{5}$ RTI International, Research Triangle Park, North Carolina, USA

\section{Correspondence to} Dr Kurt M Ribisl, Health Behavior, UNC Gillings School of Global Public Health, Chapel Hill, NC 27599, USA; kurt_ribis|@unc.edu

Received 28 January 2019 Revised 4 February 2019 Accepted 11 February 2019
Check for updates

(C) Author(s) (or their employer(s)) 2020. No commercial re-use. See rights and permissions. Published by BMJ.

To cite: Ribisl KM Chaloupka FJ, Kirchner TR, et al. Tob Control 2020;29:s27-s34.

\section{ABSTRACT}

The PhenX (Phenotypes and eXposures) Toolkit provides researchers with recommended standard consensus measures for use in epidemiological, biomedical, clinical and translational studies. To expand the depth and breadth of measures in the PhenX Toolkit, the National Institutes of Health and U.S. Food and Drug Administration have launched a project to identify 'Core' and 'Specialty' collections of measures recommended for human subjects studies in tobacco regulatory research (TRR). The current paper addresses the PhenX Toolkit TRR Vector specialty area and describes the 6-month process to identify high-priority, low-burden, scientifically supported consensus measures. Selfreported, interviewer-administered and observational measurements were considered, and input from the research community assisted in justifying the inclusion of 13 tobacco industry-relevant measures (mainly interviewer-administered or self-reported measures) in the PhenX Toolkit. Compared with measures of addiction or the use of tobacco products, assessments of many Vector factors are much newer and at an earlier stage of development. More work is needed to refine and validate measures of the spatial distribution of tobacco retailers, retail environment, price promotions and corporate social responsibility.

\section{INTRODUCTION}

The Host (eg, tobacco user), Agent (eg, tobacco product), Vector (eg, tobacco industry) and Environment (eg, policy, media)—or HAVE—model presents a useful framework to help understand and reduce tobacco use. In this model, the Vector includes the corporations that produce and market cigarettes, electronic cigarettes (e-cigarettes), cigars, pipes and smokeless tobacco; their strategies to persuade users and potential users; and their distribution systems. In 2014, the major tobacco companies spent almost US\$9.1 billion on advertising and promotions in the USA,${ }^{12}$ most of which was spent at retail outlets. In 2002, the National Tobacco Monitoring, Research and Evaluation Workshop (sponsored by the National Cancer Institute, the Centers for Disease Control and Prevention [CDC], the Robert Wood Johnson Foundation and the American Legacy Foundation [now known as the Truth Initiative]) identified numerous priority indicators of the Vector domain: tobacco brand pricing strategies, retail environment advertising and promotional allowances, grey market or smuggling activities, lobbying, direct mail marketing, tobacco brand placements in films, Internet promotions and sponsorship at bars and events. $\mathrm{Cruz}^{3}$ reviewed and identified data sources and gaps for these priority indicators and 12 other indicators of interest. In total, 38 commercial data sites, Internet sources and individual research efforts addressed the priority indicators. Unfortunately, these sources were not integrated, often costly and limited in standardisation. Cruz suggested that tobacco marketing could be more effectively monitored through the development of a national research network and called for more scientific knowledge concerning the reliability and validity of vector measures, greater standardisation of assessment methods, increased connections across studies and greater accessibility of existing data. However, despite the early interest in this area, relatively few standardised measures of Vector constructs have been published in the scientific literature. ${ }^{4}$

Following the HAVE model definition, the U.S. Food and Drug Administration (FDA) and National Institutes of Health (NIH) partnered to prioritise recommended measures for use in tobacco regulatory research (TRR), for dissemination through the PhenX (consensus measures for Phenotypes and eXposures) initiative. ${ }^{5}$ The PhenX TRR Vector Working Group (WG) was assembled to identify tobacco industry and retailer measures (ie, marketing, packaging, minors' access and retailer density) related to state, local and federal regulation of tobacco products. Between July 2014 and December 2014, WG members completed their review of and recommendations for measures and protocols for the PhenX Toolkit, a repository of recommended consensus measures available online https://www.phenxtoolkit.org/. These measures are meant to serve as a curated set of standards for cross-study analysis.

\section{METHODS \\ Developing the scope of the Vector collection}

The PhenX TRR Panel (TRRP) developed the initial scope (ie, characterising a group of measures and conditions that include similar assessments and concepts), which provided the Vector WG with a working draft to start the consensus process. The scope included 12 elements (box 1) for the Vector WG members to refer to in determining potential measures for inclusion in the PhenX Toolkit and gave them the freedom to modify the included elements based on relevance, the availability of existing protocols and other selection criteria. ${ }^{6}$ Given the concern about the number of elements to 


\section{Box 1 Initial vector scope}

- Advertising

- Affiliate marketing

- Campaign contributions and third party activities

- Exposure to smoking in the media

- Industry and retailer public relations

- Minors access

- Number, type and location of retailers

- Price and purchase location

- Product availability

- Product placement

- Promotions

- Sponsorships

cover, the Vector WG ultimately asked the Environment WG to address the minors access element.

\section{Expertise of the Vector WG}

Vector WG members were selected based on their experience in the development, evaluation and use of Vector measures in studies investigating the sales and marketing of tobacco products, tobacco control policies, tobacco industry public relations, and social and environmental determinants of tobacco product use. The WG consisted of two co-chairs, four content experts and two TRRP liaisons. Vector specialty area experts from the FDA and NIH also participated in the WG's deliberations and meetings. A complete list of WG members is provided in the Collaborators byline. In addition, the WG members consulted with numerous specialty area experts regarding the availability and selection of specific protocols. Each WG member was assigned to review two to three scope elements to identify possible measures for inclusion in the Toolkit.

\section{Complementary measures already in the PhenX Toolkit}

Over 366 measures existed in the entire PhenX Toolkit when the Vector WG was assembled. One criterion of the PhenX process is that PhenX measures must not be redundant with one another. Therefore, during their review, WG members identified measures that were already in the PhenX Toolkit that covered their assigned element(s); two existing PhenX measures were identified. Both were from the Substance Abuse and Addiction Collection (table 1). As these measures are within the scope of TRR Vector research, investigators should consider them as a complement to the recommended Vector Specialty Collection.

\section{WG deliberations}

Between July 2014 and December 2014, WG members completed their review of and recommendations for measures and protocols for the PhenX Toolkit. PhenX staff kept the Vector WG informed of potentially overlapping measures proposed by other TRR WGs. For example, an extended conversation between

\begin{tabular}{ll}
\hline $\begin{array}{l}\text { Table } 1 \\
\text { Toolkit }\end{array}$ & Complementary vector measures already in the PhenX \\
\hline PhenX measure (PhenX id) & PhenX Domain/Collection \\
\hline $\begin{array}{l}\text { Point of Sale Environment for Alcohol and } \\
\text { Tobacco (550800) }\end{array}$ & SAA (Collection) \\
\hline Cigarette Price (550101) & SAA (Collection) \\
\hline
\end{tabular}

SAA, Substance Abuse and Addiction. the Vector and Agent WG members was required to determine which WG should review the measure(s) relating to the tobacco product packaging element. The result was that the element was split, where the Agent WG would assess warning label awareness and exposure (ie, tobacco users assessing the effectiveness of health warnings on tobacco packages), and the Vector WG would focus on assessing packaging (ie, whether the tobacco products themselves are licit [compliant with tax and other policies] or illicit [not compliant with tobacco tax policies and other tobacco product regulations and/or policies]). ${ }^{7}$

In the case of the promotions element, the Vector WG agreed that their proposed Use of a Regular Brand of Tobacco measure did not compete with the Agent WG's Tobacco Brand and Variety measure. That is, the Agent measure captures the brand name and type most often used by the respondent, whereas the Vector measure only determines whether a respondent has a regular brand of tobacco. To be used effectively, both measures require incorporation of the proposed Vector measure Use of Tobacco Products.

\section{Measures for community outreach}

The measures initially selected by the WG were disseminated to the scientific community for review and feedback on 3-22 December 2014, prior to finalising the content of the TRR Vector Specialty Collection. This outreach process allowed the WG to obtain feedback from registered PhenX Toolkit users, subject matter experts and groups identified by the WG members as potential users.

Five people responded to the outreach email with general comments about some or all of the proposed measures. Four respondents completed the values section of the measures table by entering ' $\mathrm{Y}$ ' if the measure appeared to be useful or ' $\mathrm{N}$ ' if the measure did not appear to be useful. A total of 69 people visited the page on the PhenX portal that included the full data sheets for each measure and protocol. Those who responded to outreach recommended that specific instructions be added to encourage investigators to include questions about novel tobacco products, including a recommendation to consider defining 'tobacco products' as either including or excluding non-tobacco-containing nicotine or nicotine-free products. The final WG recommendation allows the user to make a determination on whether to define tobacco products as containing nicotine or not, which would generally be guided by the study purpose. (Some refillable or pod-based e-cigarette devices maynot contain nicotine e-liquids when initially sold, but are still defined astobacco products under US federal law because consumers may potentially usethem with tobacco-derived e-liquids.) A final recommendation was to broaden the definition of e-cigarettes to include other electronic nicotine delivery systems. The WG adopted these recommendations and included these details in the Specific Instructions for several measures.

\section{RESULTS}

The WG identified 13 measures for inclusion in the PhenX Toolkit and 1 measure for inclusion in the Supplemental Information (SI) section of the TRR Collections (https://www.phenxtoolkit.org/collections/supplemental/3). Table 2 lists 13 unique measures with a total of 24 protocols that meet the measurement needs of different tobacco industry and retailer measures. These unique measures refer to both measures that assess actual tobacco industry marketing practices (eg, price promotions assessed by store observations) and survey items that assess individual exposure to marketing (eg, self-reported exposure to 
Table 2 Thirteen Vector measures recommended and approved for inclusion in the PhenX Toolkit

\begin{tabular}{|c|c|}
\hline Measure name (PhenX id) & Description of measurement protocol \\
\hline $\begin{array}{l}\text { Exposure to Tobacco } \\
\text { Marketing through Internet } \\
\text { and Social Media Use } \\
(740100)^{*}\end{array}$ & $\begin{array}{l}\text { Items assess exposure and frequency of exposure to } \\
\text { tobacco marketing on the Internet and social media }\end{array}$ \\
\hline $\begin{array}{l}\text { Illicit Tobacco Products } \\
(740200)\end{array}$ & $\begin{array}{l}\text { Items identify illicit tobacco products and measure } \\
\text { the extent of illicit tobacco products in the market }\end{array}$ \\
\hline $\begin{array}{l}\text { Self-reported Exposure to and } \\
\text { Use of Price Promotions for } \\
\text { Tobacco Products (740400) }\end{array}$ & $\begin{array}{l}\text { Protocol includes } 14 \text { questions to measure consume } \\
\text { exposure to tobacco product price promotions }\end{array}$ \\
\hline $\begin{array}{l}\text { Self-reported Exposure to } \\
\text { Tobacco Advertising at Point } \\
\text { of Sale }(740500)\end{array}$ & $\begin{array}{l}\text { Protocol assesses consumer exposure to tobacco } \\
\text { product advertisements }\end{array}$ \\
\hline
\end{tabular}

Self-reported Exposure

to Tobacco Product

Advertisements (740600)

Protocol assesses tobacco product advertising and marketing exposure in adult and youth populations through traditional channels, such as magazines, television and radio

Self-reported Exposure to Questions assess exposure to tobacco product Tobacco Product Sponsorships sponsorships

(740700)

Self-reported Tobacco Product Questions assess the price paid for cigarettes, cigar, Price Paid (740800)* e-cigarettes and/or smokeless tobacco

Self-reported Tobacco Product Questions assess the purchase location of cigarettes, Purchase Location (740900) cigars, dissolvables, e-cigarettes, hookah, pipe and/or smokeless tobacco

Standardized Tobacco Observational assessment characterises the Assessment for Retail Settings availability, placement, promotion and price of (STARS) (741000) tobacco products in retail environments (eg, convenience stores, gas stations, supermarkets, pharmacies)

Tobacco Industry and Retailer 11 -item observational instrument assesses retailer Public Relations (741100) compliance with signage policies related to tobacco sales to minors and tobacco industry public relations signage in the retail sector

Tobacco Retailer Density/ Proximity $(741200)$ Protocol uses geographic information system data to determine retailer density and proximity to respondent's residence, administrative unit (census tract or block group) or school/workplace

Use of a Regular Brand of Tobacco (741300) Questions assess the respondent's regular tobacco brand for cigarettes, e-cigarettes, cigars, pipe tobacco, hookah and shisha tobacco, smokeless tobacco and dissolvable tobacco

Use of Tobacco Products Questions assess the respondent's use and regularity $(741400)+\quad$ of use of cigarettes, e-cigarettes, cigars, pipe tobacco, hookah and shisha tobacco, smokeless tobacco and dissolvable tobacco

*Selected for inclusion in the TRR Core Tier 2 Collection.

tSelected for inclusion in the TRR Core Tier 1 Collection. ${ }^{6}$

price promotions). The WG decided to include observational protocols when capturing data on retailer compliance with tobacco policies and tobacco product advertising, availability, promotions and price. Store observation (also called store audit) measures are typically conducted by one or two trained data collectors visiting tobacco retailers using a standardised audit form completed electronically or with paper and pencil. ${ }^{8}$ The WG proposed using secondary data to determine the tobacco retailer density and proximity to the respondent's residence, administrative unit or school/workplace. In contrast, selfreported and interviewer-administered protocols were identified to evaluate the respondent's opinions of the tobacco industry, purchase location and exposures to tobacco product advertisements, sponsorships and price promotions.
Each of the 13 recommended measures in the TRR Vector Specialty Collection is described below, including a brief description for each measurement protocol. Additional references to published articles and manuals of procedures for the protocols are provided under 'General References' for each measure in the PhenX Toolkit. The WG recommends that when investigators ask questions about e-cigarettes or electronic nicotine delivery systems, they depict specific examples of devices of interest and clarify various attributes. For instance, when developing a store observation form or asking about participants' exposure to e-cigarette marketing, it may make sense to separate 'cigalike' devices from more advanced systems (eg, tank systems, pod systems). They may also clarify whether the item refers to e-hookah and/ or e-cigars. As new products emerge, investigators will need to consider the inclusion of new types of products.

\section{Exposure to Tobacco Marketing through Internet and Social Media Use (PhenX id=740100)}

The WG recommended an instrument to assess exposure to tobacco marketing through new media channels, such as the Internet and social media. Items that assess exposure to tobacco marketing on the Internet and in social media were selected from the 2012 National Youth Tobacco Survey ${ }^{9}$ and the 2013 Population Assessment of Tobacco and Health (PATH) Study Wave 1 Youth Module. ${ }^{10}$ These items assess opportunities for exposure and frequency of exposure to tobacco marketing through tobacco-related advertisements, promotions and videos via text message, email, Internet and social network postings. Although the nine items may be asked of all respondents, some focus on individuals who are 12 years of age and older. The items are available in both English and Spanish and can be either self-administered or interviewer administered. The WG has provided specific instructions on the PhenX Toolkit website to further refine the administration, definition and product types addressed by these items.

\section{Illicit Tobacco Products (PhenX id=740200)}

To address the product availability element, the WG recommended an interviewer-administered instrument to assess where tobacco products are purchased and observe whether the packaging contains tax stamps and health warning labels. The protocol involves a visual inspection of the package by the interviewer. Information gathered by this instrument is used to identify illicit tobacco products (ie, those for which all relevant tobacco taxes have not been paid and/or which are not compliant with other tobacco product regulations and policies [eg, labelling and packaging policies]).

The protocol includes questions from the Pricing Policies and Control of Tobacco in Europe survey instrument. ${ }^{11}{ }^{12}$ A pack of cigarettes, for example, is identified as illicit if it has at least one of the following tax avoidance or evasion indicators: (1) it was purchased from a source that does not include all applicable taxes in the user's location (eg, a lower tax jurisdiction, such as a neighbouring state or Native American reservation), as reported by smokers; (2) it has an inappropriate tax stamp (eg, from a location other than the user's location); (3) it has an inappropriate health warning (eg, one different from the labels employed in the user's location); or (4) its price was substantially below the known price in the user's market (eg, $20 \%$ or more below the average local price). The WG has provided specific instructions on the PhenX Toolkit website to further refine the administration, definition and product types addressed by the 
recommended items. The items are appropriate for use with youth (12-17 years of age) and adults (18 years and older).

\section{Self-reported Exposure to and Use of Price Promotions for Tobacco Products (PhenX id=740400)}

The recommended interviewer-administered instrument is used to assess exposure to tobacco product price promotions, including (but not limited to) lower-price or free tobacco products, discounted prices when buying multiple tobacco product units and free or discounted tobacco product with purchase of tobacco products. This protocol includes 14 questions taken from a combination of the Wave 1 Adult and Youth PATH Survey instruments. ${ }^{10}$ The items are appropriate for use in youth (12-17 years) and adults (18 years and older) and are available in English and Spanish. The WG has provided specific instructions on the PhenX Toolkit website to further refine the administration, definition and product types addressed by the items. To answer the price promotion items, investigators will need to first determine whether a respondent has a regular brand of tobacco. Thus, the WG recommended that investigators interested in using this measure also incorporate the Use of a Regular Brand of Tobacco measure (PhenX id=741300).

\section{Self-reported Exposure to Tobacco Advertising at Point of Sale (PhenX id $=740500$ )}

The recommended interviewer-administered instrument assesses exposure to tobacco product advertising at the point of sale, including branded signs, displays, shelving units and functional items, such as trashcans and ashtrays, which have been proven to increase the appeal of and demand for tobacco products. ${ }^{13-15}$ This protocol uses a combination of questions from the Wave 1 Adult and Wave 2 Youth PATH Survey instruments. ${ }^{10}$ The items are appropriate for use in youth (12-17 years) and adults (18 years and older) and are available in English and Spanish. The WG has provided specific instructions on the PhenX Toolkit website to further refine the administration, definition and product types addressed by the items.

\section{Self-reported Exposure to Tobacco Product Advertisements (PhenX id=740600)}

The recommended protocol is an interviewer-administered instrument that measures exposure to tobacco product advertising and marketing exposure via traditional channels, such as magazines, television and radio. ${ }^{16}{ }^{17}$ This protocol uses a combination of questions from the 2011 International Tobacco Control (ITC) 4-Country Survey ${ }^{18}$ and PATH Study ${ }^{10}$ instruments. The ITC 4-Country Survey is an international cohort study of tobacco use whose overall objective is to measure the psychosocial and behavioural impact of key national-level policies. This protocol has been used in more than 20 countries.

Along with wording changes, the WG recommended that investigators should provide the respondent with advertisement images as prompts. Although PATH items were extracted from the PATH Youth Survey, the WG noted that the questions are also appropriate for adults (18 years and older). The ITC protocol is available in several languages (Bengali, Dutch, English, French, German, Hindi, Irish, Korean, Malay, Portuguese, Spanish, Thai, Dzongkha and Maori).

\section{Self-reported Exposure to Tobacco Product Sponsorships (PhenX id=740700)}

The recommended interviewer-administered survey assesses exposure to recent events sponsored by the tobacco industry.
The three questions from the ITC 4-Country Survey ${ }^{18}$ assess exposure to tobacco product sponsorships at sporting events or music, theatre, art or fashion events. The items are appropriate for youth (12-17 years) and adults (18 years and older) and are available in several languages (Bengali, Dutch, English, French, German, Hindi, Irish, Korean, Malay, Portuguese, Spanish, Thai, Dzongkha and Maori).

Along with some wording changes to the original ITC items, the WG also recommended that the interviewer indicate to the respondent that sponsored events may be identified by tobacco brand names (eg, Marlboro, Kool, Newport, Winston, Camel) or corporate names (eg, R. J. Reynolds, Philip Morris, Inc.).

\section{Self-reported Tobacco Product Price Paid (PhenX id=740800)}

The purpose of this interviewer-administered measure is to assess the prices paid for cigarettes, cigars, e-cigarettes and smokeless tobacco and how price affects consumer behaviour. Separate protocols are provided for these four types of tobacco products (PhenX ids $=740801,740802,740803$ and 740804, respectively). These protocols include questions from the Wave 1 Adult PATH Survey instrument ${ }^{10}$ and are intended for use with adults 18 years and older. Questions (available in English and Spanish) assess the amount usually purchased and the price usually paid. To answer these questions, investigators will need to determine whether the respondent is a current or former cigarette user of at least 12 months. Thus, the WG recommended that investigators interested in using this measure also incorporate the Use of Tobacco Products measure (PhenX id=741400).

\section{Self-reported Tobacco Product Purchase Location (PhenX $\mathrm{id}=740900$ )}

The WG selected seven interviewer-administered protocols (one for each tobacco product type) to determine where and how tobacco products are purchased: cigarettes (PhenX $\mathrm{id}=740901$ ), cigars (PhenX id=740902), dissolvable tobacco (PhenX id=740903), e-cigarettes (PhenX id=740904), hookah tobacco (PhenX id=740905), pipe tobacco (PhenX id=740906) and smokeless tobacco (PhenX id=740907). These protocols include questions from the Wave 1 Adult PATH Survey instrument ${ }^{10}$ and are intended for use with adults 18 years and older. Three questions (available in English and Spanish) assess how the product was purchased (ie, in person, Internet, telephone or by someone else), where the product was purchased (ie, in-state, out-of-state or outside the USA) and from what type of store (eg, convenience store, supermarket). To answer these questions, investigators will need to determine whether the respondent is a current or former tobacco product user of at least 12 months. To do so, the WG recommended that investigators interested in using this measure also incorporate the Use of Tobacco Products Toolkit measure (PhenX id $=741400)$.

\section{Standardized Tobacco Assessment for Retail Settings (STARS) (PhenX id=741000)}

The WG recommended the observational assessment protocol from the STARS 1.0 surveillance tool, which was designed for investigators to inform state and local tobacco control policies for the point of sale. ${ }^{19}$ The protocol includes 17 items for an observer to complete in stores that sell tobacco products, such as convenience stores, gas stations, supermarkets and pharmacies. It was developed for the National Cancer Institute's State and Community Tobacco Control Initiative by the Retail Environment WG, with additional support from the CDC's Epidemiology Branch. The instrument and ancillary training materials are 
intended for use by self-trained youth and adults. The protocol was selected because of its reliability, user-friendly format and short completion time (it should take, on average, 9-10 min to complete).

\section{Tobacco Industry and Retailer Public Relations (PhenX id=741100)}

This 11-item instrument observational measure, taken from California's Statewide Youth Tobacco Purchase Survey (YTPS), assesses retailer compliance with signage policies related to tobacco sales to minors and tobacco industry public relations signage in the retail sector. ${ }^{2021}$ This survey collects information about minors' purchase attempts and observations of the purchasers during these attempts. For the purpose of this measure, only items within the 'Observations' section of the YTPS are used; these relate to the presence, type and location of signs stating that tobacco sales to minors are unlawful. Item number 8 of the 2014 Statewide YTPS was selected because it captures the presence and location of both public relations signage and local, state and federal signage related to tobacco purchases by minors. The instrument includes blank sections that allow for new types of signage to be recorded.

The WG has provided specific instructions to further refine the administration, definition and types of marketing addressed by these items. To capture the retail sector environment related to tobacco industry marketing more comprehensively, this measure should be used in conjunction with the STARS (PhenX $\mathrm{id}=741000)$.

\section{Tobacco Retailer Density/Proximity (PhenX id=741200)}

This recommended measure characterises the retail availability of tobacco products by quantifying the proximity and density of retailers with respect to residential locations and other points of interest (POIs), such as schools. Using geospatial data, retail 'density' measures the spatial concentration of tobacco retailers in a neighbourhood, which is defined as either an area centred on a respondent's residence or school/workplace or an administrative area, such as a county, school district or census tract. 'Proximity' measures the linear, two-dimensional distance between one or more tobacco retailer from a POI under study (eg, residence, school, other reference centroid).

Three protocols are provided in the Toolkit to assess tobacco retailer density and proximity within neighbourhood areas, such as U.S. census tracts (PhenX id=741201), relative to known residence locations (PhenX id=741202) and known school locations (PhenX id=741203). Assuming that information on a study respondent's current address (see PhenX DemographicsCurrent Address [PhenX id=010800]) has been collected, geographic address coding or 'geocoding' allows linking that address to measures of tobacco retailer proximity and density. However, valid data sources providing the locations of tobacco product retailers are required. In addition, calculating density requires a definition of a 'neighbourhood' or other spatial unit that is then linked with census-based information about neighbourhood characteristics, such as land area, roadway miles or population size. The protocols compute the density for each neighbourhood, residence or school by dividing the count of retailers by the land area (eg, retailers per square kilometre). Alternatively, the density can be computed by dividing the count of retailers by the population in a spatial unit (eg, retailers per 1000 people) or by using smoothed kernel density estimation methods. To assess density within U.S. census polygons, which is often necessary when a more specific residential location is unknown, the protocol of Duncan et al ${ }^{22}$ is provided. To assess retailer density and proximity to a known geocode for residence, the protocol of Young-Wolff et $a l^{23}$ is used. To assess the proximity to the respondent's school, the protocol of Luke et $a l^{24}$ is provided.

The Tobacco Retailer Density/Proximity measure was identified as high burden because it takes longer than $15 \mathrm{~min}$ to implement and requires knowledge of census data products and websites, such as American Factfinder (https://factfinder.census. gov/), and/or commercial geospatial data products. Calculating the recommended density/proximity measures requires the use of geographic information systems, such as ESRI ArcGIS (ESRI, Redlands, California, USA). After extracting the necessary data, statistical expertise is needed to draw appropriate inferences related to neighbourhood estimates. ${ }^{25-27}$

\section{Use of a Regular Brand of Tobacco (PhenX id=741300)}

As tobacco product brands are industry manufacturing and marketing commodities, the WG recommended assessing the use of a regular brand. The purpose of this interviewer-administered measure is to determine if a respondent has a preferred brand for any or all of the following seven tobacco product types: cigarettes, e-cigarettes, cigars, pipe tobacco, hookah and shisha tobacco, smokeless tobacco and dissolvable tobacco. ${ }^{10}$ It is intended for use in adults 18 years of age and older. To answer these questions, investigators must determine if the respondent is a current or former tobacco product user of at least 12 months by first applying the Use of Tobacco Products measure (PhenX $\mathrm{id}=741400)$.

\section{Use of Tobacco Products (PhenX id=741400)}

The purpose of this measure is to assess a respondent's use and regularity of use of the following tobacco products: cigarettes, e-cigarettes, cigars, pipe tobacco, hookah and shisha tobacco, smokeless tobacco and dissolvable tobacco. This intervieweradministered protocol includes questions from the Wave 1 Adult PATH Survey instrument and the PATH Wave 1 Individual Screener. It is intended for use in adults 18 years of age and older. ${ }^{10}$ Analysing the results allows respondents to be categorised as ever user, current user, everyday user, some-day user, 30-day user, experimental current user, experimental former user, former user, former user 12 months or former user 12 months plus. Images of exemplar product types are included, along with product-specific questions.

\section{Measures not selected for inclusion}

Although measures for the campaign contributions element were identified and considered valuable, the WG agreed that more systematic monitoring and analysis are needed. Therefore, none of the identified instruments were determined to be suitable for inclusion in the Toolkit. In addition, the WG decided that the exposure to smoking in the media/product placement element would be better addressed by the Environment $\mathrm{WG}^{28}$ because it was considered an environmental factor (ie, the media environment) that may influence tobacco use. The Public Opinion of the Tobacco Industry measure was chosen by the WG for inclusion in the SI. This recommendation was made for two reasons: First, no analysis of the data obtained using the protocol via the California Department of Public Health's Media Tracking Survey had been published. Second, other measures were considered higher priority for inclusion in the Toolkit.

\section{DISCUSSION}

The primary goal of the Vector WG was to identify validated instruments that focus on the sales and marketing practices of 
the tobacco industry. In some cases, the instruments focus on tobacco products and packaging; advertising and marketing strategies through print, media and retail channels; and product pricing and taxation. In other instances, the focus was on how the consumer, who is often a tobacco user, recalled seeing tobacco advertising or where they purchased their tobacco products and how much they paid for them. Overall, the modes of assessment included self-reported surveys of tobacco users, observations of where tobacco products are sold and marketed and inspections of tobacco product packaging.

The Vector measures are designed to capture what are among the most potent factors affecting tobacco use. Tobacco product prices, which are strongly related to excise tax levels, are perhaps the strongest factor affecting tobacco use. ${ }^{29}$ Pricereducing promotions, such as US\$1.00-off discounts on a pack of cigarettes, stimulate consumption, and according to the Federal Trade Commission, ${ }^{12}$ tobacco companies spend most of their advertising and marketing budget on such promotions. We also know that the role of 'place' looms large over tobacco control, ${ }^{30}$ including the targeted distribution and pricing of products within neighbourhood environments. ${ }^{31}$ Studies have shown that greater retailer density near schools is related to higher youth smoking rates ${ }^{32}$ and that the close proximity of tobacco retail outlets undermines smoking cessation attitudes and behaviour. ${ }^{33-35}$

Compared with measures of addiction or use of tobacco products, many Vector measures are at an earlier stage of development. With few exceptions, US states have not systematically surveyed the tobacco retail environment until recently. Henriksen and colleagues ${ }^{19}$ found that 42 of the 50 states surveyed were now conducting some form of store audit, with the STARS being the most commonly used. Municipal governments are also beginning to implement passive surveillance systems that use comprehensive photographic data collection and machine learning analytics that can rapidly and repeatedly assess street-level conditions, including the operational status and advertising practices of retail outlets. ${ }^{36-38}$ As surveillance systems continue to evolve, tobacco vector surveillance efforts may benefit from strategic national partnerships, such as the one between the FDA youth sales inspection programme and the Substance Abuse and Mental Health Services Administration's Synar programme, ${ }^{39}$ both of which coordinate tens of thousands of unannounced outlet inspections annually across all 50 states. ${ }^{4041}$ Moreover, these partnerships can help update existing Vector surveillance systems or fill in gaps (eg, campaign contributions).

Understanding the impact of the tobacco retail environment on tobacco use, including exposure to retail marketing (eg, quantity of advertising, price and promotions, marketing of flavours and alternative tobacco products) and spatial access to tobacco products (eg, tobacco retailer density), is an area that is ripe for the development of additional Vector measures. Surveillance of exposure to tobacco marketing on the Internet and social media is an emerging area in tobacco control research requiring new measures to complement self-reported assessments on US surveys. In addition, the Vector WG noted a dearth of measures on consumer knowledge of and attitudes about tobacco companies. Such measures would facilitate gauging the effect of anti-industry messaging, such as the corrective statements ordered by the US Federal Court, ${ }^{42}$ and pro-industry messaging, such as corporate social responsibility marketing. ${ }^{43}$

Many countries have adopted stronger restrictions on tobacco marketing than the USA, and some Vector measures in the PhenX Toolkit are included in routine international surveillance instruments, such as the Global Youth and Adult
Tobacco Surveys and surveys by the ITC Policy Evaluation Project in 29 countries. ${ }^{44}$ Even the price of the cheapest cigarette pack from the STARS instrument can be compared with data collected in urban and rural neighbourhoods across lowincome, middle-income and high-income countries using the Environmental Profile of a Community's Health (EPOCH), which assesses environmental determinants of cardiovascular health. ${ }^{45}$ In addition, one adaptation of a widely used ITC scale $^{46}$ has incorporated information about the retail availability of tobacco from EPOCH community assessments. ${ }^{45}$

The number of US state and local jurisdictions that restrict tobacco marketing (eg, eliminating price promotions, such as coupons and discounts, and sales of flavoured tobacco products) is increasing. ${ }^{47}$ In this rapidly changing policy context, comparative measures will be needed, such as the strength of tobacco control scores or percentage of the population covered by strong policies. Lessons from global tobacco control suggest that implementation assessment (ie, 'policies on the street') will be as important as legal research (ie, 'policies on the books'). ${ }^{45}$

In closing, the Vector WG identified 13 measures assessing tobacco industry factors that will inform TRR. The consistent use of these measures in the PhenX Toolkit will advance regulatory science by encouraging scientists to use consistent, validated measures, which will allow comparisons across studies and the aggregation of data sets.

What this paper adds

- The sales and marketing practices of the tobacco industry are among the most potent factors affecting the initiation and maintenance of tobacco product use. Industry practices include product packaging; advertising and marketing strategies through print, media and retail channels; and product pricing and taxation. The reliable assessment of these practices and consumer perceptions is central to identifying risk factors for tobacco use and for regulatory considerations.

- The continuous proliferation of novel tobacco products as advertised and marketed across a variety of platforms introduces challenges for scientists seeking to support evidence-based regulatory decision-making because suitable measures may not be readily available or widely known.

- The Vector Working Group made consensus recommendations to investigators in the field concerning the best methods to assess exposure to tobacco marketing through Internet and social media use and through traditional channels, such as magazines, television and radio; illicit tobacco products; price promotions; advertising at the point of sale; product sponsorships; and actual prices paid. Methods to assess product purchase locations, including retail settings, retailer density and proximity to schools, and retailer compliance with local and state signage policies were also included in the Toolkit.

- As tobacco regulatory science evolves, it will be critical for investigators to consistently use valid and comprehensive measures of multiple sources of tobacco industry and retailer practices and their influence on use.

Acknowledgements We gratefully acknowledge the contributions of the anonymous members of the scientific community who commented on the measures proposed for inclusion. We also acknowledge the contribution of Deborah Maiese, RTI International, who led the recruitment of WG members and the orientation to the PhenX process, and the contributions of the RTI editorial team, including Michelle 
Bogus, Judy Cannada, Loraine Monroe and Amy Morrow, for providing expert editorial review of the manuscript.

Collaborators The following are members of the PhenX Tobacco Regulatory Research (TRR) Vector Working Group (WG): Co-Chairs Frank J Chaloupka, University of Illinois at Chicago, and Kurt Ribisl, University of North Carolina at Chapel Hill; Vector WG members Lisa Henriksen, Stanford Prevention Research Center, Tom R Kirchner, New York University, Ruth Malone, University of California, San Francisco, and Rachel Grana Mayne, National Cancer Institute; TRR Panel Liaisons Andrew Hyland, Roswell Park Comprehensive Cancer Center, and Kay L Wanke, NIH Office of Disease Prevention; and the PhenX team from RTI International, including Tabitha Hendershot, RTI TRR Project Lead, Destiney S Nettles, WG Supervisor, and Rebecca Geisler, WG manager.

Contributors GES, TPH and KLW led the conceptualisation of the manuscript and the coordination of the writing team. KMR and GES drafted the scientific content of the manuscript, and DSN and TPH drafted the Methods and Results sections of the manuscript. TRK and KLW reviewed the draft and provided substantive revisions. FJC, LH and RGM reviewed the draft and provided comments. The Co-Chairs and Members of the PhenX TRR Vector WG identified and proposed preliminary measures and voted on final measures included in the PhenX TRR Vector Specialty Collection. TRRP members AH and KLW ensured that the WG process maintained fidelity with overall project goals. Federal Agency Liaison Katherine A Margolis, FDA Center for Tobacco Products, ensured project consistency with agency goals and priorities. NIH Project Coordinator KLW proposed the PhenX TRR initiative and contributed to its execution and completion. The PhenX team coordinated and facilitated the WG process, including project oversight and leadership (TPH), supervisory management (DSN) and project management (RG). PhenX NIH Program Official Erin M Ramos, National Human Genome Research Institute, provided project guidance and funding coordination. Carol M Hamilton, RTI International, is the PhenX Principal Investigator and provided project guidance and supervision.

Funding Research reported in this publication was supported by grant numbers U41HG007050 from the National Human Genome Research Institute (NHGRI) and U41HG007050-01S1 from the NHGRI and FDA Center for Tobacco Products (CTP).

Disclaimer The content is solely the responsibility of the authors and does not necessarily represent the official views of the $\mathrm{NIH}$ or the FDA.

Competing interests Dr. Ribisl serves as an expert consultant in litigation against tobacco companies. No other interests were declared.

Patient consent for publication Not required.

Provenance and peer review Not commissioned; internally peer reviewed.

\section{REFERENCES}

1 Federal Trade Commission. Cigarette report for 2014. Washington, DC: Federal Trade Commission, 2016.

2 Federal Trade Commission. Smokeless tobacco report for 2014. Washington, DC: Federal Trade Commission, 2016

3 Cruz TB. Monitoring the tobacco use epidemic IV. The vector: tobacco industry data sources and recommendations for research and evaluation. Prev Med 2009;48(1 Suppl):S24-S34.

4 Garcia-Cazarin ML, Mandal RJ, Grana R, et al. Host-agent-vector-environment measures for electronic cigarette research used in NIH grants. Tob Control 2019;28:s181-7.

5 Maiese DR, Hendershot T, Strader L, et al. PhenX-establishing a consensus process to select common measures for collaborative research. Research Triangle Park, NC: RTI Press, 2013.

6 Swan GE, Hendershot TP, van Bemmel DM, et al. The PhenX toolkit: core measures for tobacco regulatory research. Tob Control 2019;28:s139-42.

7 O'Connor RJ, Watson CH, Swan GE, et al. PhenX: agent measures for tobacco regulatory research. Tob Control 2019;28:s158-64.

8 Lee JGL, Henriksen L, Myers AE, et al. A systematic review of store audit methods for assessing tobacco marketing and products at the point of sale. Tob Control 2014;23:98-106.

9 Centers for Disease Control and Prevention (CDC), National Youth Tobacco Survey (NYTS). Secondary National Youth tobacco Survey (NYTS). Available: www.cdc.gov/ tobacco/data_statistics/surveys/nyts/ [Accessed 28 Jan 2019].

10 U.S. Department of Health and Human Services, National Institutes of Health, National Institute on Drug Abuse. Population Assessment of Tobacco and Health (PATH) Study [United States] Restricted-Use Files. Ann Arbor, MI: Inter-university Consortium for Political and Social Research [distributor], 2018.

11 Gallus S, Lugo A, La Vecchia C, et al. Pricing policies and control of tobacco in Europe (PPACTE), WP2: European survey on smoking. Dublin, Ireland: PPACTE Consortium, 2012.

12 Joossens L, Lugo A, La Vecchia C, et al. Illicit cigarettes and hand-rolled tobacco in 18 European countries: a cross-sectional survey. Tob Control 2014;23:e17-23.
13 Paynter J, Edwards R. The impact of tobacco promotion at the point of sale: a systematic review. Nicotine Tob Res 2009;11:25-35.

14 Robertson L, McGee R, Marsh L, et al. A systematic review on the impact of point-ofsale tobacco promotion on smoking. Nicotine Tob Res 2015;17:2-17.

15 Robertson L, Cameron C, McGee R, et al. Point-of-sale tobacco promotion and youth smoking: a meta-analysis. Tob Control 2016;25:e83-9.

16 International Agency for Research on Cancer.. IARC handbooks of cancer prevention, volume 12: methods for evaluating tobacco control policies. Lyon, France: International Agency for Research on Cancer, 2008.

17 Farrelly MC, Healton CG, Davis KC, et al. Getting to the truth: evaluating national tobacco countermarketing campaigns. Am J Public Health 2002;92:901-7.

18 ITC Policy Evaluation Project. International tobacco control 4-Country survey 2011, 2011. Available: https://www.itcproject.org/surveys [Accessed 28 Jan 2019].

19 Henriksen L, Ribisl KM, Rogers T, et al. Standardized Tobacco Assessment for Retail Settings (STARS): dissemination and implementation research. Tob Control 2016;25(Suppl 1):i67-74.

20 Apollonio DE, Malone RE. The "We Card" program: tobacco industry "youth smoking prevention" as industry self-preservation. Am J Public Health 2010;100:1188-201.

21 California Department of Public Health ", California Tobacco Control Program. Statewide youth tobacco purchase survey 2014. Section II, observations, item 8 Sacramento, CA: California Department of Public Health, California Tobacco Control Program, 2014

22 Duncan DT, Kawachi I, Subramanian SV, et al. Examination of how neighborhood definition influences measurements of youths' access to tobacco retailers: a methodological note on spatial misclassification. Am J Epidemiol 2014;179:373-81.

23 Young-Wolff KC, Henriksen L, Delucchi K, et al. Tobacco retailer proximity and density and nicotine dependence among smokers with serious mental illness. Am J Public Health 2014;104:1454-63.

24 Luke DA, Ribisl KM, Smith C, et al. Family Smoking Prevention and Tobacco Control Act: banning outdoor tobacco advertising near schools and playgrounds. Am J Prev Med 2011;40:295-302.

25 Frank LD, Schmid TL, Sallis JF, et al. Linking objectively measured physical activity with objectively measured urban form: findings from SMARTRAQ. Am J Prev Med 2005;28(2 Suppl 2):117-25.

26 Timperio A, Crawford D, Telford A, et al. Perceptions about the local neighborhood and walking and cycling among children. Prev Med 2004;38:39-47.

27 Colabianchi N, Dowda M, Pfeiffer KA, et al. Towards an understanding of salient neighborhood boundaries: adolescent reports of an easy walking distance and convenient driving distance. Int J Behav Nutr Phys Act 2007;4.

28 Unger JB, Chaloupka FJ, Vallone D, et al. PhenX TRR environment Working group. Tob Control 2019;28:s173-80.

29 Chaloupka FJ, Yurekli A, Fong GT. Tobacco taxes as a tobacco control strategy. Tob Control 2012;21:172-80.

30 Koh H. Place matters for tobacco control. JAMA 2016;316:700-1.

31 National Cancer Institute. The role of the media in promoting and reducing tobacco use. Bethesda, MD: U.S. Department of Health and Human Services, National Institutes of Health, National Cancer Institute, 2008.

32 Henriksen L, Feighery EC, Schleicher NC, et al. Is adolescent smoking related to the density and proximity of tobacco outlets and retail cigarette advertising near schools? Prev Med 2008:47:210-4

33 Cantrell J, Anesetti-Rothermel A, Pearson JL, et al. The impact of the tobacco retail outlet environment on adult cessation and differences by neighborhood poverty. Addiction 2015;110:152-61.

34 Kirchner TR, Cantrell J, Anesetti-Rothermel A, et al. Geospatial exposure to pointof-sale tobacco: real-time craving and smoking-cessation outcomes. Am J Prev Med 2013;45:379-85

35 Reitzel LR, Cromley EK, Li Y, et al. The effect of tobacco outlet density and proximity on smoking cessation. Am J Public Health 2011;101:315-20.

36 Ilakkuvan V, Tacelosky M, Ivey KC, et al. Cameras for public health surveillance: a methods protocol for crowdsourced annotation of point-of-sale photographs. JMIR Res Protoc 2014;3:e22.

37 Cantrell J, Ganz O, llakkuvan V, et al. Implementation of a multimodal mobile system for point-of-sale surveillance: lessons learned from case studies in Washington, DC, and New York City. JMIR Public Health Surveill 2015;1.

38 Ganz O, Cantrell J, Moon-Howard J, et al. Electronic cigarette advertising at the point-of-sale: a gap in tobacco control research. Tob Control 2015;24:e110-2.

39 Substance Abuse and Mental Health Services Administration, US Food and Drug Administration. A strategic partnership, 2010. Available: http://www.fda.gov/ downloads/TobaccoProducts/NewsEvents/UCM284343. pdf [Accessed 28 Jan 2019].

40 U.S. Department of Health and Human Services. Food and Drug Administration. Enforcement action plan for promotion and advertising restrictions. $21 \mathrm{cfr}$ part 1140, 2010. Available: https://www.fda.gov/TobaccoProducts/GuidanceComplian ceRegulatorylnformation/ucm271744.htm [Accessed 28 Jan 2019].

41 Kirchner TR, Villanti AC, Tacelosky M, et al. National enforcement of the FSPTCA at point-of-sale. Tob Regul Sci 2015;1:24-35.

42 Cappella JN, Maloney E, Ophir Y, et al. Interventions to correct misinformation about tobacco products. Tob Regul Sci 2015;1:186-97. 
43 Epperson AE, Prochaska JJ, Henriksen L. The FLIP side of natural American spirit: corporate social responsibility advertising. Tob Control 2018; 27:355-6.

44 Henriksen L. Comprehensive tobacco marketing restrictions: promotion, packaging, price and place. Tob Control 2012;21:147-53.

45 Chow CK, Corsi DJ, Gilmore AB, et al. Tobacco control environment: cross-sectional survey of policy implementation, social unacceptability, knowledge of tobacco health harms and relationship to quit ratio in 17 low-income, middle-income and high-income countries. BMJ Open 2017;7:e013817.

46 Joossens L, Raw M. The tobacco control scale: a new scale to measure country activity. Tob Control 2006;15:247-53.

47 Lange T, Hoefges M, Ribisl KM. Regulating tobacco product advertising and promotions in the retail environment: a roadmap for states and localities. J Law Med Ethics 2015;43:878-96. 